\title{
Proteinogenic Amino Acid Assisted Preparation of Highly Luminescent Hybrid Perovskite Nanoparticles
}

Anna Jancik Prochazkova, ${ }^{1,2}$ Stepan Demchyshyn, ${ }^{1,3}$ Cigdem Yumusak, ${ }^{1}$ Jiř́i Másilko, ${ }^{2}$ Oliver Brüggemann, ${ }^{4}$ Martin Weiter, ${ }^{2}$ Martin Kaltenbrunner, ${ }^{3}$ Niyazi Serdar Sariciftci, ${ }^{1}$ Jozef Krajcovic ${ }^{2}$, Yolanda Salinas, ${ }^{4}$ and Alexander Kovalenko ${ }^{1,2^{*}}$

${ }^{1}$ Linz Institute for Organic Solar Cells (LIOS), Physical Chemistry, Johannes Kepler University Linz, Altenbergerstraße 69, 4040 Linz, Austria

${ }^{2}$ Faculty of Chemistry, Materials Research Centre, Brno University of Technology, Purkyňova 118, 61200 Brno, Czech Republic

${ }^{3}$ Soft Electronics Laboratory, Linz Institute of Technology (LIT), Johannes Kepler University Linz, Altenbergerstraße 69, 4040 Linz, Austria

${ }^{4}$ Institute of Polymer Chemistry (ICP), Johannes Kepler University Linz, Altenbergerstraße 69, 4040 Linz, Austria

Corresponding author:

E-mail: kovalenko.alx@gmail.com

Keywords: lead halide perovskites, nanoparticles, amino acids, ligands, colloids, luminescence

\begin{abstract}
Versatile approaches to nanoparticle synthesis offer unprecedented opportunities for the development of optoelectronics, photonics, as well as bio sciences. With the current advancement of hybrid organic-inorganic metal halide perovskites, the next step is to expand their field of applications via utilization of functional and modifiable ligand chemistry. Here, we present a ligand assisted reprecipitation approach for highly luminescent perovskite nanoparticle synthesis using for the first time L-lysine and L-arginine for surface passivation. These nanoparticles exhibit emission within a narrow bandwidth of the visible spectrum and photoluminescence quantum yield close to $100 \%$. Additionally, preferential ligand orientation is achieved via amino acids $\alpha$-amino group blocking which results in blueshifted emission as well as smaller and more uniform particle size. These experimental results demonstrate the effectiveness of naturally occurring proteinogenic amino acids as surface ligands and offer possibilities for versatile modification of perovskite nanoparticle properties via well-studied amino acid chemistry.
\end{abstract}

\section{Introduction}

Lead halide perovskites have ushered a new era in the field of electronics and photonics. In addition to the superior performance in photovoltaic cells, ${ }^{1-8}$ perovskites are successfully implemented in solid state lasers, ${ }^{9}$ light emitting diodes, ${ }^{10}$ photodetectors ${ }^{11}$, and solar fuel production, ${ }^{12}$ which is possible due to their high-absorption coefficient and long-ranged ambipolar transport. Besides thin film and single crystal devices, ${ }^{13-16}$ perovskite nanoparticles (PNP) are of great interest owing their high photoluminescence quantum yield (PLQY) and optical tenability. PNP are prospective active materials for light emitting diodes (LEDs), ${ }^{17}$ photodetectors ${ }^{18}$ and lasers. ${ }^{19}$ The main advantage of 
perovskites over classic inorganic semiconductors is their low-temperature, low-cost solution based deposition on a large variety of the substrates via coating or printing techniques. ${ }^{20-22}$ One of the most significant issues in perovskite thin film production is that the final material is synthetized in situ, from its precursors. This formation process places high demand on the processing conditions, which can result in significant increase of the final cost of the product and hinder the mass production of perovskite-based devices. Films produced from a stable dispersion of PNP can resolve this issue.

One of the key aspects to PNP synthesis is crystal growth termination through surface ligands, which are essential components of nucleation and growth processes, thus effecting material stability and PLQY. Long alkyl chains are commonly used as surface ligands, ${ }^{23,24}$ however the perovskite nanoparticles stabilized by multidentate polymer micelles ${ }^{25}$, conjugated ligands ${ }^{26}$ and cysteine ${ }^{27}$ were also reported. CdSe and $\mathrm{ZnS}$, conventional semiconductor quantum dots, become water soluble and biocompatible when decorated with appropriate cysteine and hystydine ligands. ${ }^{28}$ Recently reported work describe perovskite films cross-linked with bi-functional 5-aminovaleric acid ${ }^{29}$ and perovskite nanoparticles passivated with peptide chains of various length for potential photovoltaic and optoelectronic application. ${ }^{30}$ In this paper we would like to demonstrate the next step towards combining the unique properties of PNP such as high absorption coefficient, high PLQY and charge carrier transport with properties of amino acids (AAs)/peptides such as affinity to the desired surfaces, self-assembly through the weak interactions and sensing abilities.

Here, we introduce proteinogenic essential AAs L-lysine (LYS) and L-arginine (ARG) as effective ligands for PNP synthesis. Comparative measurements of PNP preparation procedures using these AAs in combination with different solvents, and stabilizing carboxylic acids illustrate the intricate nature of the synthesis route. Another key point is the ability of amino acids to self-organize through the weak interaction, which acts as a useful tool for the PNP preparation. In the present paper we focus on the preserving amino acid functional group, thus it is described the possibility to block the amino group and selectively passivate the surface of the perovskite nanoparticles in a manner that primary amine or guanidyl group is bonded to the surface of PNP, and the $\alpha$-amino acid group is directed outwards. Therefore, in order to reveal the practicality of AA chemistry, we blocked the $\alpha-$ amino group with tert-butyloxycarbonyl ( $t$ Boc) protecting group, which serves a twofold purpose: (i) selective orientation of the AAs ligand due to selective bonding of the positively charged R-groups with the core of the nanoparticle and (ii) possible further functionalization of the PNP via deprotection and modification of $\alpha$-amino group that now are preferentially exposed to additional reactants. The synthesis route utilizing $t$ BOC modified AAs results in PNP with diameters $<6 \mathrm{~nm}$, a small size distribution, and bright blue emission.

\section{Experimental}

Perovskite NP preparation. PNP were prepared by the ligand assisted reprecipitation method, according to González-Carrero et al. ${ }^{31}$. Alongside with LYS and ARG ligands, hexanoic acid (HEA) was chosen as the primary stabilizing carboxylic acid. Additionally, oleic (OLA) and adamantanecarboxylic (ADA) acids were used to test what influence long/bulk chain carboxylic acids exert on the formation of nanoparticles. Additionally, we attempted to synthesize PNP using acetic acid as a stabilizer; however no colloidal dispersion was obtained, which assumingly occurred due to high hydroscopicity of acetic acid, thus excessive water in the precursor solution hindered the formation of PNP. For precipitation, toluene (Tol) and chloroform (Chl) were used as antisolvent and dispersion media. As the result, 4 main samples using HEA and 8 supplementary samples using OLA and ADA were prepared. 


\section{Results and Discussion}
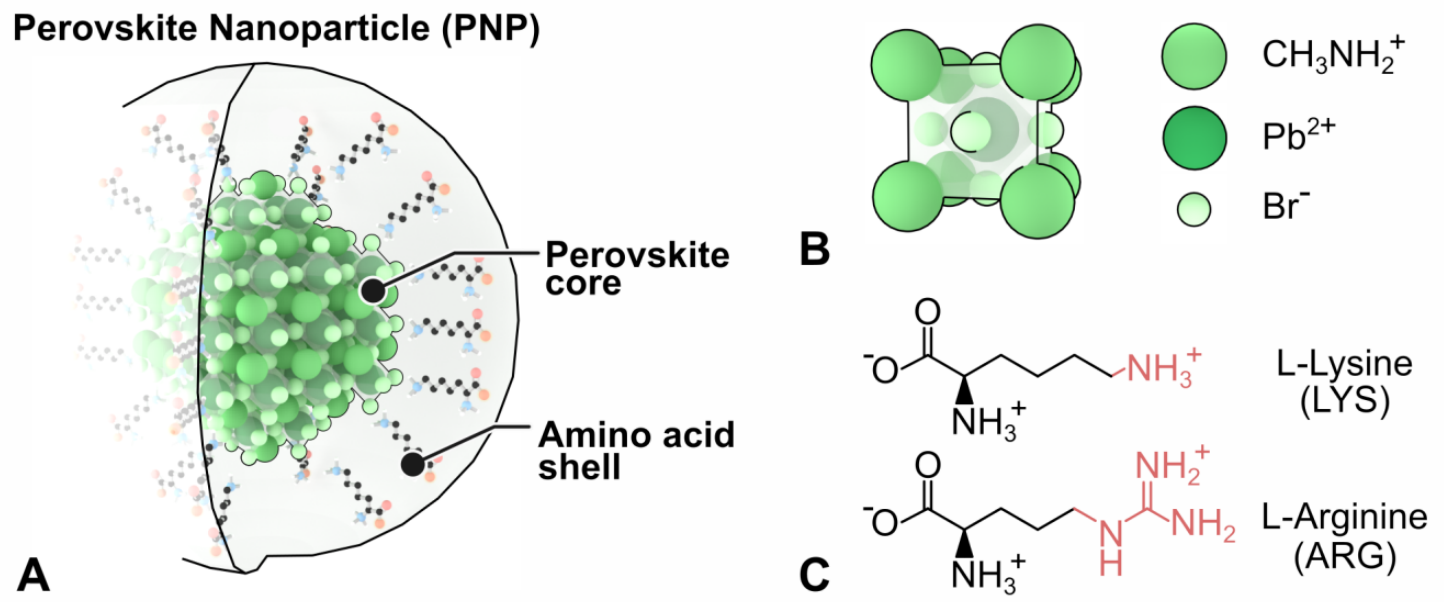

Figure 1. $\mathrm{MAPbBr}_{3}$ PNP capped with AA ligands. (A) Schematic representation of PNP. (B) Crystalline structure of $\mathrm{MAPbBr}_{3}$, the core material of PNP. (C) Chemical structure of LYS and ARG, proteinogenic amino acids with positively charged R-groups (highlighted in red: secondary amino group (LYS) and guanidine group (ARG)).

Precursor solution was prepared by dissolving $\mathrm{MABr}(0.150 \mathrm{mmol}), \mathrm{PbBr}_{2}(0.136 \mathrm{mmol})$ relevant amino acid $(0.109 \mathrm{mmol})$ and carboxylic acid $(1.30 \mathrm{mmol})$ in $2.5 \mathrm{~mL}$ of DMF, at room temperature. The molar ratio of chemicals was 1:1.1:0.8:9.5 for $\mathrm{PbBr}_{2}, \mathrm{MABr}$, amino acid and carboxylic acid, respectively. Consequently, the solution was sonicated in an ultrasonic bath at room temperature for $10 \mathrm{~min}$ and then was left to be magnetically stirred overnight. For the precipitation of PNP the vial with chloroform/toluene was placed in the ice bath at $0.3-1.0{ }^{\circ} \mathrm{C}$ with a magnetic stirrer. Then $20 \mu \mathrm{L}$ of the precursor solution were added dropwise to the vial with chloroform/toluene. To adjust the concentration of the PNP in the solvent, the volume of toluene/chloroform was filled to total $5 / 10 / 25 \mathrm{~mL}$. Similarly, to the previously reported work on perovskite nanoparticles no further purification step was applied. ${ }^{1}$ It has to be noted, that other conventional solvents were used for the PNP precipitation, such as: heptane, acetone and diethylether. However, none of the above-mentioned dispersion media resulted in green-emitting colloidal suspension.

In order to obtain solid material for FTIR and powder XRD characterization, colloidal solutions were prepared by precipitation of $1 \mathrm{~mL}$ of precursor solution in $50 \mathrm{~mL}$ of solvent in an ice bath, followed by centrifugation ( $5000 \mathrm{rpm}$ for $20 \mathrm{~min}$ ). Supernatant was discarded and the solid material was dried in a vacuum oven $\left(40^{\circ} \mathrm{C}\right)$ overnight. 


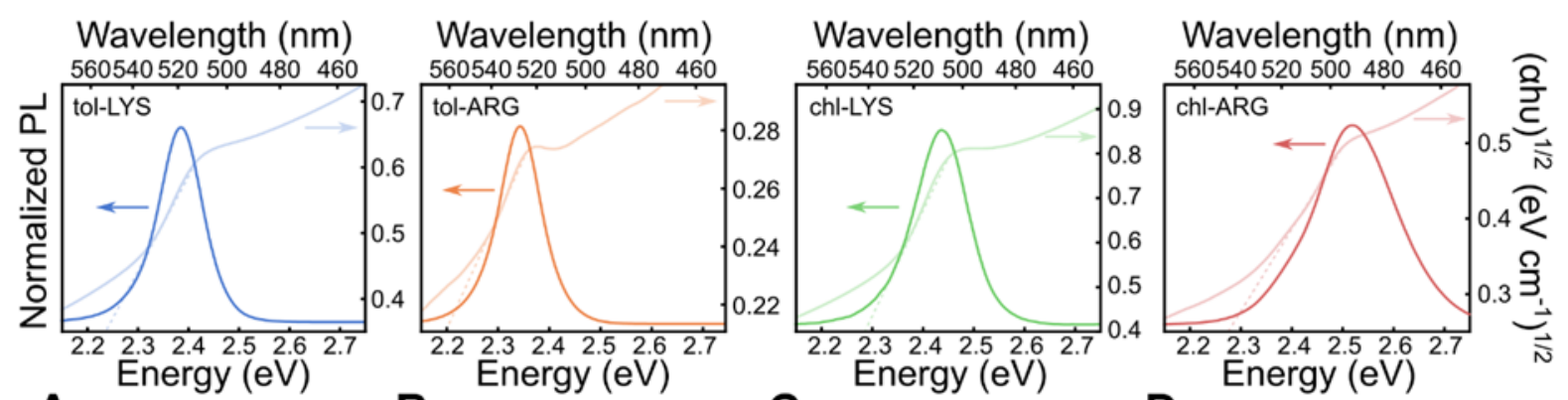

A

B

C

D

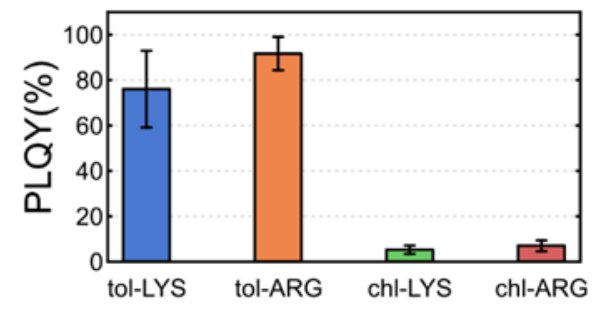

E

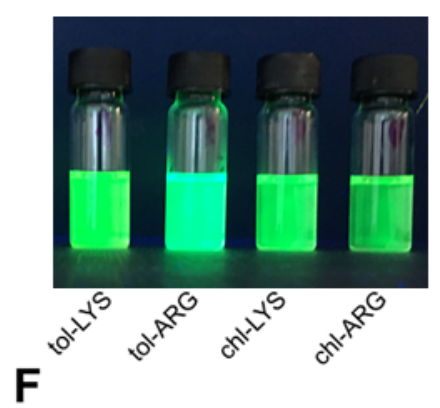

Figure 2. Optical data of the colloidal PNP. PL spectra and Tauc absorption plots of (A) LYS and (B) ARG capped PNP dispersed in toluene compared to (C) LYS and (D) ARG capped PNP dispersed in chloroform. Samples dispersed in toluene show redshifted emission $\left(\lambda_{\operatorname{MAX}}(\right.$ Tol-LYS $)=520 \mathrm{~nm}$, $\lambda_{\text {MAX }}($ Tol-ARG $\left.)=528 \mathrm{~nm}\right)$ when compared to the samples dispersed in chloroform $\left(\lambda_{\text {MAX }}(\right.$ Chl-LYS $)$ $=509 \mathrm{~nm}, \lambda_{\operatorname{MAX}}($ Chl-LYS) $=492 \mathrm{~nm}$ ) (optical data summarized in Table 1). (E) Comparison of PLQY of the PNP prepared in different solvents and ligands, with $95 \%$ confidence intervals. Samples prepared in toluene show significantly higher PLQY than those prepared in chloroform. (F) Photographs of the corresponding PNP suspensions under UV illumination.

Here we present perovskite nanoparticles (PNP) synthesized using surface ligands comprising of naturally occurring proteinogenic amino acids (AAs) (Fig. 1A). The PNP consist of a methyl ammonium lead bromide $\left(\mathrm{MAPbBr}_{3}\right)$ core known for its excellent optical properties ${ }^{32,33}$ (Fig. 1B) capped with a shell of L isomers of LYS or ARG (Fig. 1C). The zwitterionic nature of these AAs, i.e. the presence of both negatively and positively charged groups in one molecule, allows for the passivation of $\mathrm{Pb}^{2+}$ and excess $\mathrm{Br}^{-}$defects on the surface of the PNP, therefore increasing the effectiveness of ligand passivation. ${ }^{27}$ Hexanoic acid (HEA) used as a stabilizing component serves the role of a protonation agent, thus increasing the amount of charged groups present in the solution. ${ }^{34}$ Photoluminescence (PL) and absorption spectra of $\mathrm{MAPbBr}_{3}$ PNP colloid prepared with the abovementioned components in toluene (Fig. 2A, B) and chloroform (Fig. 2C, D) show strong emission and an absorption onset in the green part of the visible spectrum. Toluene based colloids exhibit red shifted emission at $\lambda_{\operatorname{MAX}}($ Tol-LYS $)=520 \mathrm{~nm}$ and $\lambda_{\operatorname{MAX}}($ Tol-ARG $)=528 \mathrm{~nm}$, whereas PNP dispersed in chloroform show emission at higher energy, $\lambda_{\mathrm{MAX}}(\mathrm{Chl}-\mathrm{LYS})=509 \mathrm{~nm}$ and $\lambda_{\mathrm{MAX}}$ $(\mathrm{Chl}-\mathrm{ARG})=492 \mathrm{~nm}$ (complete optical data summarized in Tab. 1). PNP prepared in toluene exhibit an order of magnitude higher PLQY $(\eta($ Tol-LYS $)=76 \pm 17 \%, \eta($ Tol-ARG $)=92 \pm 17 \%)$ when compared to the complementary samples prepared in chloroform ( $\eta(\mathrm{Chl}-\mathrm{LYS})=5 \pm 2 \%, \eta(\mathrm{Chl}-\mathrm{ARG})$ $=7 \pm 2 \%$ ) (Fig. 2E). The different optical behavior of the PNP in these solvents can be attributed to electronegative chlorine groups in $\mathrm{CHCl}_{3}$, that also influence PNP synthesis by coordination with $\mathrm{Pb}^{2+} \cdot 35,36$ Additionally, lower PLQY in chloroform samples might be the direct outcome of the aprotic nature of the solvent that often acts as luminescence quencher ${ }^{37,38}$. To test this assumption, we add an 
equal amount of toluene to all the samples prepared in chloroform and vice versa. As a result, addition of chloroform to toluene-based samples results in an average five-fold PLQY decrease from initial $\sim 100 \%$ to $\sim 20 \%$. On the other hand, the addition of toluene to chloroform-based samples yields an approximately two-fold PLQY increase. The bright green luminescence (Fig. 2F) of the colloidal suspensions in toluene remained stable over the period of one week, retaining $>80 \%$ of the initial PLQY values, whereas chloroform based counterparts lost most of their luminescence in the same period of time. Notably, during the experiments no visible sedimentation was observed.
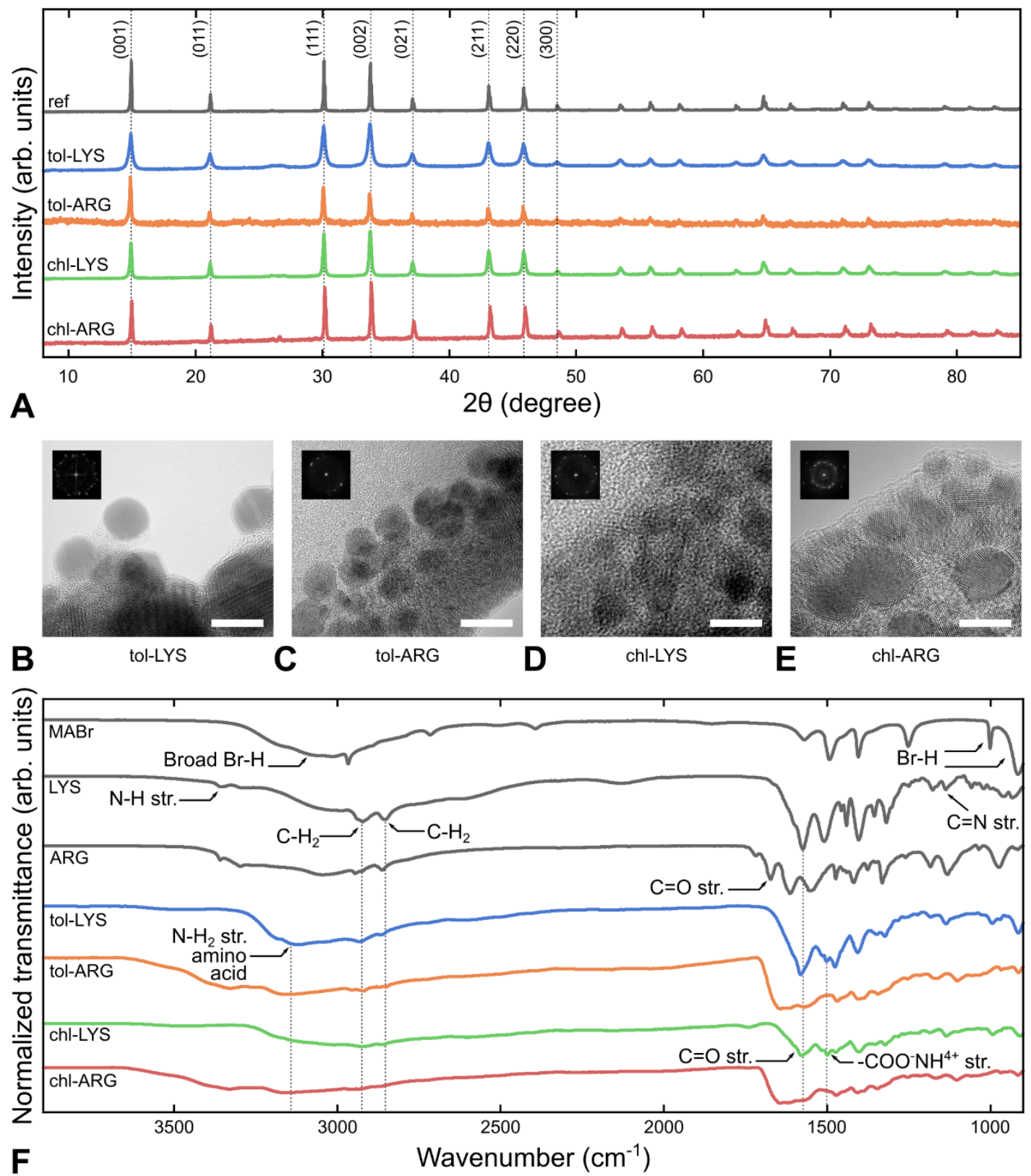

Figure 3. Structural analysis of PNP capped with amino acids. (A) Powder XRD analysis of samples precipitated from toluene (blue, orange) and chloroform (green, red) PNP suspensions. Diffractograms show characteristic peaks that can be directly compared to $\mathrm{MAPbBr}_{3}$ reference (gray). (B-E) TEM images of corresponding PNP (scale bar $5 \mathrm{~nm}$ ) with FFT pattern inset (additional 
information on size and distribution shown in Table 1). (F) FTIR spectra show typical absorption peaks that are attributed to the presence of AA ligands on the surface of PNP.

Corresponding measurements were performed for the samples prepared with OLA and ADA as stabilizing acid (Fig. S1A). The peak PL emission is in the same range as in HEA samples (Fig. S1B), whereas the full width at half maximum (FWHM) tends to be lower in colloids containing ADA (Fig. S1C). This can be attributed to a higher degree of self-organization that is common in adamantyl compounds. $^{39,40}$

Table 1: Summary of optical data obtained from spectroscopic experiments as well as PNP average size measured from TEM images. CI - $95 \%$ confidence interval.

\begin{tabular}{|l|c|c|c|c|c|c|}
\hline $\begin{array}{l}\text { Sample } \\
\text { label }\end{array}$ & $\begin{array}{l}\text { Stabilizing } \\
\text { acid }\end{array}$ & $\lambda_{\max }(\mathbf{n m}) /(\mathbf{e V})$ & $\begin{array}{l}\text { FWHM (nm)/ } \\
\mathbf{( e V )}\end{array}$ & $\begin{array}{l}\text { PLQY } \pm \\
\mathbf{C I}(\%)\end{array}$ & $\mathbf{E}_{\mathbf{g}}(\mathbf{e V})$ & $\begin{array}{l}\text { PNP diameter } \pm \\
\text { CI (nm) }\end{array}$ \\
\hline Tol-LYS & HEA & $520 / 2.4$ & $22 / 0.1$ & $76 \pm 17$ & 2.0 & $9.3 \pm 0.9$ \\
\hline Tol-ARG & HEA & $528 / 2.3$ & $22 / 0.1$ & $92 \pm 7$ & 1.7 & $6.9 \pm 0.4$ \\
\hline Chl-LYS & HEA & $509 / 2.4$ & $26 / 0.1$ & $5 \pm 2$ & 2.1 & $5.5 \pm 0.3$ \\
\hline Chl-ARG & HEA & $492 / 2.5$ & $36 / 0.2$ & $7 \pm 2$ & 1.9 & $6.4 \pm 0.3$ \\
\hline
\end{tabular}

Where: $\lambda_{\max }$ - wavelength at the emission maximum, FWHM - full width at half maximum of the emission peak; $E_{\mathrm{g}}$ - optical bandgap.

Structural characterization. In order to further investigate the material's properties, PNP were deposited onto a solid support by centrifugation from colloidal dispersion, followed by vacuum drying. Using powder XRD we confirm the perovskite crystalline structure by comparing PNP diffractograms to bulk $\mathrm{MAPbBr}_{3}$ diffraction patterns available in the literature ${ }^{41}$ (Fig. 3A). The peaks positioned at $14.90^{\circ}, 21,17^{\circ}, 26,01^{\circ}, 30,13^{\circ}, 33,80^{\circ}, 37,10^{\circ}, 43,09^{\circ}$, and $45,88^{\circ}$ correspond to the reflections (001), (011), (111), (002), (021), (211), (220), and (300), respectively. These results are comparable to previously published literature ${ }^{14,42}$ and confirm the PNP cubic unit cell structure. A broad peak at around $26^{\circ}$ is observed in Tol-LYS samples and can be attributed to incomplete crystallization influenced by HEA as stabilizing agent. When replaced with ADA, two narrow peaks appear in the same position, which suggests that the ability of ADA to self-organize improves PNP crystallinity (Fig. S2A). PNP samples in general exhibit peak broadening when compared to bulk $\mathrm{MAPbBr}_{3}$ that stems from the small size of the crystallites. However, in the present case this is not a quantitative method for the size determination since AAs tend to decompose under X-ray irradiation 43,44. PNP size analysis was performed via transmission electron microscopy (TEM) (Fig. 3B-E). In general, highly crystalline PNP with diameters $<10 \mathrm{~nm}$ are observed in all the samples (Tab. 1). Samples prepared with OLA and ADA result in PNP with a slightly smaller diameter that is on average $<9 \mathrm{~nm}$ (Fig.S 2B-I, Tab. S1). It was observed that the smallest PNP were formed when adamantanecarboxylic acid was used, as confirmed by TEM analysis. However these samples didn't show a significant difference in the optical gap. Thus it can be assumed, that in case, when adamantanecarboxylic acid is used as a stabilizer, the shell of the nanoparticles is smaller as compared to the other samples. Regarding the precipitation medium, samples prepared in chloroform possessed wider bandgap, thus the emission maxima were blue-shifted. This phenomenon can be related to the interaction of electronegative chlorine groups in $\mathrm{CHCl}_{3}$ with perovskite precursors. It has to be noted, that when no amino acid was added in the precursor solution, after the precipitation immediate 
sedimentation of an orange solid was observed. TEM images revealed relatively large cubic crystallites were observed (see SI).

Fourier transform infrared (FT-IR) spectroscopy is used to detect the presence of LYS and ARG capping ligands on the surface of the PNP (Fig. 2F). MABr has three main characteristic peaks at $2700-3300 \mathrm{~cm}^{-1}$ (broad signal) assigned to $\mathrm{Br}-\mathrm{H}$ and two more signals at around 1000 (narrow) and at $912 \mathrm{~cm}^{-1}$ (medium) assigned to the $\mathrm{C}-\mathrm{H}$ rocking bonds ${ }^{45}$. Those signals were detected in all prepared PNP, which can be an indication of residual MABr traces, since no further purification was perfomed after PNP preparation. However, those bands can also be attributed to the amino groups from the AAs, which are also visible in all the PNP spectra. The shift of $\mathrm{C}=\mathrm{O}$ vibration from AA carboxylic group of LYS (at $1577 \mathrm{~cm}^{-1}$ ) or ARG (at $1672 \mathrm{~cm}^{-1}$ ) appears at higher wavenumbers in Tol-LYSADA $\left(1584 \mathrm{~cm}^{-1}\right)$ and lower in Chl-ARG-ADA sample $\left(1639 \mathrm{~cm}^{-1}\right)$ respectively. Furthermore, the peak in the region around $1498 \mathrm{~cm}^{-1}$ is assigned to the $-\mathrm{COONH}_{3}{ }^{+}$group from the carboxylic acid incorporation, in agreement with Ebrahiminezhad et al. ${ }^{46}$. Generally, pure LYS and ARG show two bands at 2851 and $2925 \mathrm{~cm}^{-1}$ attributed to the asymmetric and symmetric $\mathrm{CH}_{2}$ stretching, respectively, signals confirmed in both materials tol-LYS and chl-LYS spectra. Additionally, as expected, $\mathrm{C}-\mathrm{N}$ stretching and amine groups from the AAs were succesfullly detected at $1181 \mathrm{~cm}^{-1}$ and $3150 \mathrm{~cm}^{-1}$ in both LYS and ARG -based PNP spectra. ${ }^{47}$ 


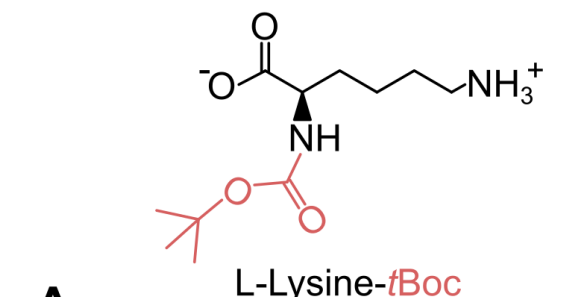

A
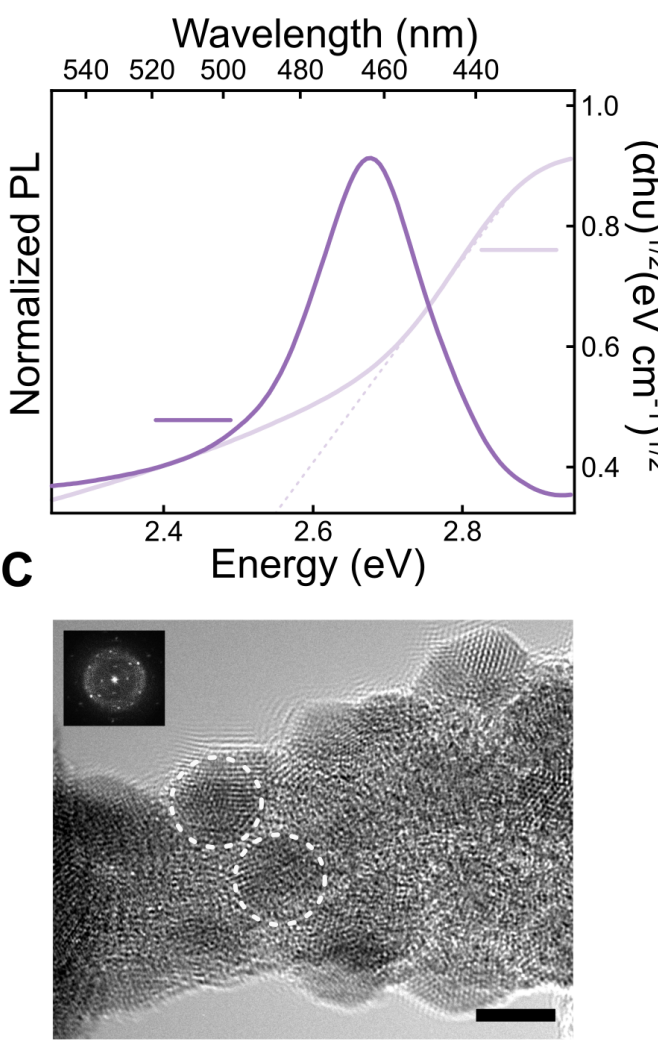

$\mathbf{E}$

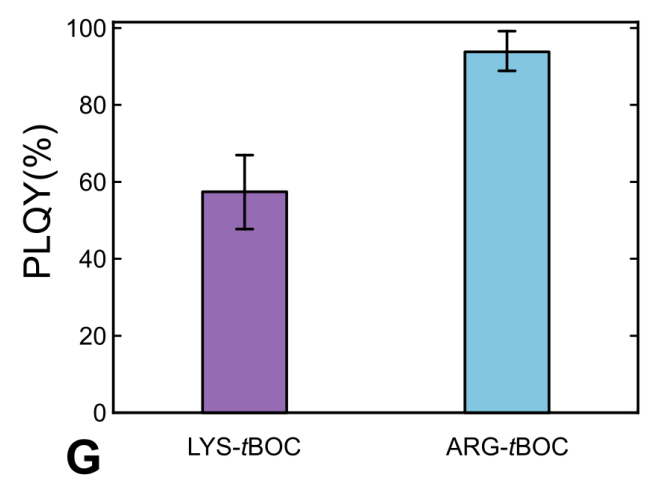<smiles>CC(C)(C)OC(=O)N[C@@H](CCCNC(N)=[NH2+])C(=O)[O-]</smiles>

B

L-Arginine-tBoc
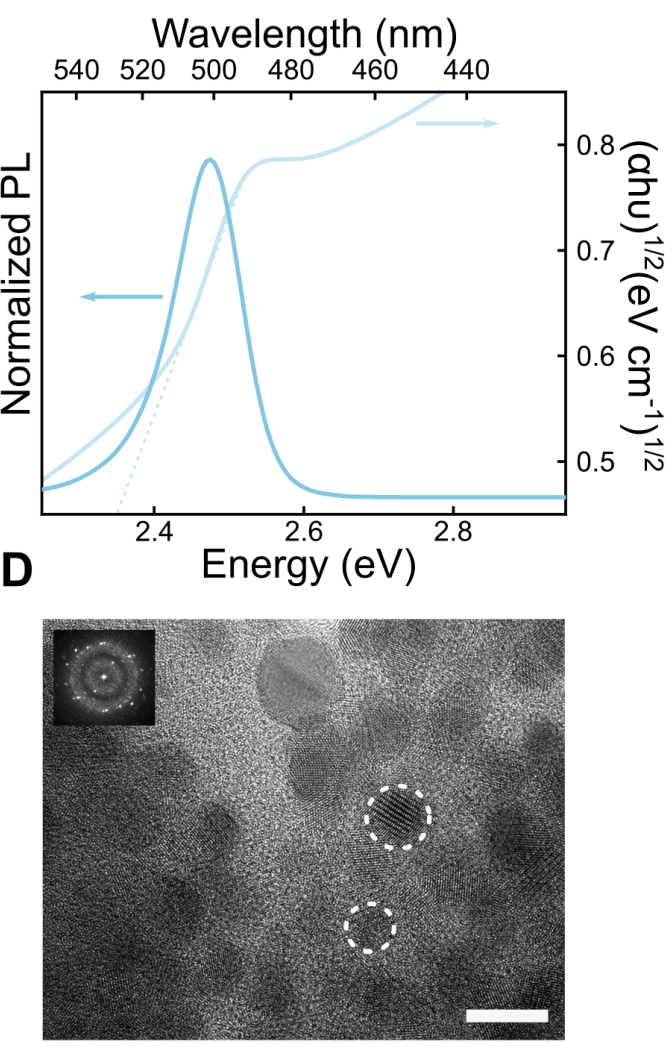

$\mathbf{F}$

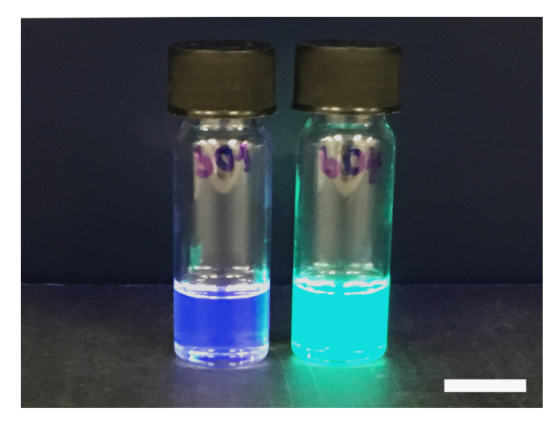

H

Figure 4: Characterization of PNP capped with modified amino acids. Chemical structures of (A) LYS and (B) ARG modified with $t$ BOC group at their $\alpha$-amino group position (highlighted in red) in order to promote selective bonding to the PNP perovskite core. PL spectra and Tauc absorption plots of (C) LYS-tBOC and (D) ARG- $t$ BOC. PL emission of $t$ BOC modified samples $\left(\lambda_{\text {MAX }}\right.$ (LYS- $t$ Boc $)=$ $463 \mathrm{~nm}, \lambda_{\mathrm{MAX}}($ ARG- $t$ Boc $\left.)=501 \mathrm{~nm}\right)$ appear to be blue shifted $\left(\Delta_{\mathrm{LYS}}=57 \mathrm{~nm}\right.$ and $\left.\Delta_{\mathrm{ARG}}=27 \mathrm{~nm}\right)$ when compared to their unmodified counterparts. (optical data summarized in Table S2_. TEM images and FFT data (insets) of precipitated (E) LYS- $t$ BOC (scale bar $=5 \mathrm{~nm}$ ) and (F) ARG- $t$ BOC 
$($ scale bar $=10 \mathrm{~nm})$. The images clearly show formation of nanoparticles (highlighted with dashed lines) with an average diameter of $\mathrm{d}($ LYS- $t$ Boc $)=4.4 \pm 0.3 \mathrm{~nm}$ and $\mathrm{d}(\mathrm{ARG}-t$ Boc $)=5.7 \pm 0.3 \mathrm{~nm}$. (G) PLQY results for LYS- $t$ Boc and ARG- $t$ Boc samples, with $95 \%$ confidence interval error bars. (H) Photographs of LYS- $t$ Boc and ARG-tBoc PNP suspensions under UV illumination show bright blue and cyan luminescence visible to the naked eye (scale bar $-1 \mathrm{~cm})$.

ARG and LYS amino acids are known to self-organize through the weak interaction between deprotonated carboxyl and the $\alpha$-amino group or between carboxyl and amine (LYS)/guanidine (ARG) in R-group. ${ }^{48,}{ }^{49}$ (Fig. S3) Additionally, there are two possibilities for PNP stabilization with positively charged AA functional groups: interaction between $\mathrm{Br}^{-}$surface defects and $\alpha$-amino groups or amine (LYS)/guanidine (ARG). In order to force selective bonding between positively charged Rgroups of the AAs and $\mathrm{Br}^{-}$PNP defects, $\alpha$-amino group of LYS and ARG are protected with $t$ Boc group (Fig. 4A, B). This prompts passivation via amine (LYS)/guanidine (ARG) and leaves the $\alpha-$ amino group facing the outer surface of the PNP. This can be utilized for further functionalization of the ligands. ${ }^{50,51}$ Figure 4C, D show the spectra of PNP prepared using $\mathrm{N}_{\alpha}-t$ Boc-L-lysine (LYS- $t$ Boc) and $\mathrm{N}_{\alpha}-t$ Boc -L-arginine (ARG- $t$ Boc) in combination with hexanoic acid and dispersed in toluene. These samples result in PNP emission in blue and cyan part of the spectra, respectively ( $\lambda_{\text {MAX }}$ (LYS$t$ Boc $)=463 \mathrm{~nm}, \lambda_{\text {MAX }}($ ARG- $t$ Boc $\left.)=501 \mathrm{~nm}\right)($ complete optical data summary available in Tab. 2$)$. Additionally, the emission peak of LYS- $t$ Boc and ARG- $t$ Boc is significantly blue-shifted when compared to their unmodified counterparts $(\Delta \lambda(\mathrm{LYS})=57 \mathrm{~nm}, \Delta \lambda(\mathrm{ARG})=27 \mathrm{~nm})$. TEM images of PNP centrifuged onto a solid surface and dried under vacuum show a collection of particles with an average diameter $\mathrm{d}($ LYS $-t$ Boc $)=4.4 \pm 0.3 \mathrm{~nm}$ and $\mathrm{d}(\mathrm{ARG}-t \mathrm{Boc})=5.7 \pm 0.3 \mathrm{~nm}$. Notably, PNP prepared using modified AAs had smaller diameter, when compared to their unmodified counterparts. This can be attributed to better self-assembly and more organized bonding via amine (LYS)/guanidine (ARG) of the modified ligands, as well as by the presence of the $t$ Boc group, which prevents selforganization of the amino acids in the solution. Thus functional groups are free to interact with the perovskite precursors, resulting in more efficient PNP formation. The FFT pattern reveals the presence of a large amorphous phase fraction. We assume that the perovskite core size is below the diameter observed in TEM due to the presence of surface ligands. Taking this into consideration, the large blue shift can be partly attributed to quantum confinement effects (reported $\mathrm{MAPbBr}_{3}$ exciton Bohr radius between 2.2-3.5 $\mathrm{nm}^{52}$ ). The PLQY of the samples synthesized with modified AAs remains high, though for LYS- $t$ Boc it is at $\sim 60 \%$, whereas for ARG- $t$ Boc it is $\sim 95 \%$ (Fig. 4G). Both LYS- $t$ Boc and ARG- $t$ Boc PNP suspensions show bright blue and cyan luminescence under UV illumination visible to a naked eye (Fig. 4H).

Table 2. Summary of the optical data collected from PNP containing $t$ Boc functionalized amino acids. CI $-95 \%$ confidence interval.

\begin{tabular}{|l|l|l|l|l|l|l|}
\hline $\begin{array}{l}\text { Sample } \\
\text { label }\end{array}$ & $\begin{array}{l}\text { Stabilizing } \\
\text { acid }\end{array}$ & $\lambda_{\max }(\mathbf{n m}) /(\mathbf{e V})$ & $\begin{array}{l}\text { FWHM (nm)/ } \\
\mathbf{( e V )}\end{array}$ & $\begin{array}{l}\text { PLQY } \pm \\
\text { CI (\%) }\end{array}$ & $\mathbf{E}_{\mathbf{g}}(\mathbf{e V})$ & $\begin{array}{l}\text { PNP } \\
\text { diameter } \pm \\
\text { CI (nm) }\end{array}$ \\
\hline LYS- $t$ Boc & HEA & $463 / 2.7$ & $32 / 0.2$ & $57 \pm 10$ & 2.4 & $4.4 \pm 0.3$ \\
\hline ARG- $t$ Boc & HEA & $501 / 2.5$ & $22 / 0.1$ & $94 \pm 5$ & 2.2 & $5.7 \pm 0.3$ \\
\hline
\end{tabular}

Where: $\lambda_{\max }$ - wavelength at the emission maximum, FWHM - full width at half maximum of the emission peak; $\mathrm{E}_{\mathrm{g}}$ - optical bandgap. 
Above-mentioned approach shows a great application potential for development of the optoelectronics devices based on PNP stabilized with amino acid, mainly due to the versatility and large variety of naturally occurring amino acids and short-chain peptides. However, at the moment the biggest challenge, which hinders the implementation of these devices, is a preparation of smooth and compact layers to provide electrical characterization (i.e. charge carrier transport) of the PNP. Preliminary results, showed, that thin films, prepared by spin coating or centrifugal casting ${ }^{31}$, had excessive roughness (see figure S5) to be further implemented for the electrical characterization and device fabrication. To solve this issue a thorough investigation has to be performed. Possibly, different substrates or different surface ligands have to be used (e.g. shorter chain hydroxylic acids). Another possibility seems to be the utilization of different amino acids or short-chain peptides, which tend to self-aggregate in in a manner, that smooth and compact layers would be obtained.

\section{Conclusion}

In conclusion, we demonstrate a new and frugal approach to effectively stabilize PNP using naturally occurring proteinogenic AAs. The PNP showed bright green emission with the exact PL peak position influenced by the ligand and solvent used. Structural analysis confirmed the preservation of the core $\mathrm{MAPbBr}_{3}$ crystalline structure, as well as the presence of AAs ligands on the surface of the PNP. Particle sizes have been analyzed via TEM imagining revealing PNP diameters of $<10 \mathrm{~nm}$. AAs modified with $t \mathrm{BOC}$ group, blocking $\alpha$-amino group, allowed for preferential ligand bonding via their positively charged R-group. This resulted in more efficient PNP surface passivation, manifesting itself in strong blue shift, smaller PNP diameter, and high PLQY. Further modification of AAs ligands may open new ways for chemical functionalization finding the use in sensing, solar cells, and optoelectronics. Eventual improvement in stability of AA modified PNP will allow them to enter the field of biological applications such as imaging and assays.

\section{Acknowledgements:}

This work was supported by the Czech Science Foundation grant No. 19-23718S, by the European Research Council Starting Grant "GEL-SYS" (Grant Agreement No. 757931) and a startup grant of the Linz Institute of Technology (Grant Agreement No. LIT013144001SEL). Research infrastructure was supported by project no. REG LO1211 from the National Programme for Sustainability I (MEYS CR). Niyazi Serdar Sariciftci acknowledges financial support of the Austrian Science Foundation (FWF) [Z 222-N19] within the Wittgenstein Prize.

\section{Supporting Information.}

The Supporting Information is available free of charge on the ACS Publications website at DOI: 10.1021/acsanm.XXXXXXX.

Experimental Techniques and Materials; PNP characterization; Figure S1: Influence of stabilizing acids on optical properties of PNPs suspensions; Table S1: Summary of optical data and PNP size; Figure S2: Structural characterization of PNPs prepared with oleic and adamantanecarboxylic acid; Figure S3; Arginine amino acids bonded by weak interaction; Figure S4: TEM images of the precipitated precursor solution without amino added; Figure S5. AFM image tBoc-Lysine stabilized PNP.

\section{References:}


(1) Docampo, P.; Ball, J. M.; Darwich, M.; Eperon, G. E.; Snaith, H. J. Efficient organometal trihalide perovskite planar-heterojunction solar cells on flexible polymer substrates. Nat. Commun. 2013, 4, 2761.

(2) Hui-Seon, K.; Mora-Sero, I.; Gonzalez-Pedro, V.; Fabregat-Santiago, F.; Juarez-Perez, E. J.; Park, N.-G.; Bisquert, J. Mechanism of carrier accumulation in perovskite thin-absorber solar cells. Nat. Commun. 2013, 4, 2242.

(3) Stranks, S. D.; Eperon, G. E.; Grancini, G.; Menelaou, C.; Alcocer, M. J. P.; Leijtens, T.; Herz, L. M.; Petrozza, A.; Snaith, H. J. Electron-hole diffusion lengths exceeding 1 micrometer in an organometal trihalide perovskite absorber. Science. 2013, 342, 341-344.

(4) Mei, A.; Li, X.; Liu, L.; Ku, Z.; Liu, T.; Rong, Y.; Xu, M.; Hu, M.; Chen, J.; Yang, Y.; Grätzel, M.; Han, H.; A hole-conductor-free, fully printable mesoscopic perovskite solar cell with high stability. Science. 2014, 345, 295-298.

(5) Christians, J. A.; Fung, R. C. M.; Kamat, P. V. An inorganic hole conductor for organo-lead halide perovskite solar cells. improved hole conductivity with copper iodide. J. Am. Chem. Soc. 2014, 136, 758-764.

(6) Liu, D.; Yang, J.; Kelly, T. L. Compact layer free perovskite solar cells with $13.5 \%$ efficiency. J. Am. Chem. Soc. 2014, 136, 17116-17122.

(7) Nie, W.; Tsai, H.; Asadpour, R.; Blancon, J-C.; Neukirch, A. J.; Gupta, G.; Crochet, J. J.; Chhowalla, M.; Tretiak, S.; Alam, M. A.; Wang, H.-L., Mohite, A. D. High-efficiency solution-processed perovskite solar cells with millimeter-scale grains. Science. 2015, 347, $522-525$.

(8) Choi, J. J.; Yang, X.; Norman, Z. M.; Billinge, S. J. L.; Owen, J. S. Structure of methylammonium lead iodide within mesoporous titanium dioxide: active material in highperformance perovskite solar cells. Nano Lett. 2014, 14, 127-133.

(9) Xing, G.; Mathews, N.; Yantara, N.; Sabba, D.; Mhaisalkar, S.; Lim, S. S.; Liu, X.; Sum, T. C.; Graetzel, M. Low-temperature solution-processed wavelength-tunable perovskites for lasing. Nat. Mater. 2014, 13, 476-480.

(10) Tan, Z.-K.; Moghaddam, R. S.; Lai, M. L.; Docampo, P.; Higler, R.; Deschler, F.; Price, M.; Sadhanala, A.; Pazos, L. M.; Credgington, D.; Hanusch, F.; Bein, T.; Snaith H.; J.; Friend, R. H.; Bright light-emitting diodes based on organometal halide perovskite. Nat. Nano. 2014, 9, 687-692.

(11) Dou, L.; Yang, Y.; You, J.; Hong, Z.; Chang, W.-H.; Li, G.; Yang, Y. Solution-processed hybrid perovskite photodetectors with high detectivity. Nat. Commun. 2014, 5, 5404.

(12) Chen, Y.-S.; Manser, J. S.; Kamat, P. V. All solution-processed lead halide perovskite-BiVO 4 tandem assembly for photolytic solar fuels production. J. Am. Chem. Soc. 2015, 137, 974981.

(13) Kovalenko, A.; Pospisil, J.; Krajcovic, J.; Weiter, M.; Guerrero, A.; Garcia-Belmonte, G. Interface inductive currents and carrier injection in hybrid perovskite single crystals. Appl. Phys. Lett. 2017, 111, 163504-163504.

(14) Kovalenko, A.; Pospisil, J.; Zmeskal, O.; Krajcovic, J.; Weiter, M. Ionic origin of a negative capacitance in lead halide perovskites. Phys. Status Solidi RRL. 2017, 11, 1600418

(15) Chen, Z.; Dong, Q.; Liu, Y.; Bao, C.; Fang, Y.; Lin, Y.; Tang, S.; Wang, Q.; Xiao, X.; Bai, Y.; Deng, Y.; Huang, J.; Thin single crystal perovskite solar cells to harvest below-bandgap light absorption. Nat. Commun. 2017, 8, 1-7.

(16) Saidaminov, M. I.; Adinolfi, V.; Commin, R.; Abdelhady, A. L.; Peng, W.; Dursun, I.; Yuan, M.; Hoogland, S.; Sargent, E. H.; Bakr, O. M. Planar-integrated single-crystalline perovskite photodetectors. Nat. Commun. 2015, 6, 8724 . 
(17) Kumawat, N. K.; Gupta, D.; Kabra, D. Recent advances in metal halide-based perovskite light-emitting diodes. Energy Technol. 2017, 5, 1734-1749.

(18) Jeon, Y. P.; Woo, S. J.; Kim, T. W. Transparent and flexible photodetectors based on $\mathrm{CH}_{3} \mathrm{NH}_{3} \mathrm{PbI}_{3}$ perovskite nanoparticles. Appl. Surf. Sci. 2018, 434, 375-381.

(19) Tang, B.; Dong, H.; Sun, L.; Zheng, W.; Wang, Q.; Sun, F.; Jiang, X.; Pan, A.; Zhang, L. Single-mode lasers based on cesium lead halide perovskite submicron spheres. ACS Nano. 2017, 11, 10681-10688.

(20) Noh, J. H.; Im, S. H.; Heo, J. H.; Mandal, T. N.; Seok, S. I. Chemical management for colorful, efficient, and stable inorganic-organic hybrid nanostructured solar cells. Nano Lett. 2013, 13, 1764-1769.

(21) Filip, M. R.; Eperon, G. E.; Snaith, H. J.; Giustino, F. Steric engineering of metal-halide perovskites with tunable optical band gaps. Nat. Commun. 2014, 5, 5757.

(22) D’Innocenzo, V.; Kandada, A. R. S.; De Bastiani, M.; Gandini, M.; Petrozza, A. Tuning the light emission properties by band gap engineering in hybrid lead halide perovskite. J. Am. Chem. Soc. 2014, 136, 17730-17733.

(23) Schmidt, L. C.; Pertegas, A.; Gonzalez-Carrero, S.; Malinkiewicz, O.; Agouram, S.; Minguez Espallargas, G.; Bolink, H. J.; Galian, R. E.; Perez-Prieto, J. Nontemplate synthesis of $\mathrm{CH}_{3} \mathrm{NH}_{3} \mathrm{PbBr}_{3}$ perovskite nanoparticles. J. Am. Chem. Soc. 2014, 136, 850-853.

(24) Zhang, F.; Zhong, H.; Chen, C.; Wu, X.; Hu, X.; Huang, H.; Han, J.; Zou, B.; Dong, Y. Brightly luminescent and color-tunable colloidal $\mathrm{CH}_{3} \mathrm{NH}_{3} \mathrm{PbX}_{3}(\mathrm{X}=\mathrm{Br}, \mathrm{I}, \mathrm{Cl})$ quantum dots: Potential alternatives for display technology. ACS Nano. 2015, 9, 4533-4542.

(25) Hou, S.; Guo, Y.; Tang, Y.; Quan, Q. Synthesis and stabilization of colloidal perovskite nanocrystals by multidentate polymer micelles. ACS Appl. Mater. Interfaces. 2017, 9, $18417-$ 18422.

(26) Dai, J.; Xi, J.; Li, L.; Zhao, J. F.; Shi, Y.; Zhang, W.; Ran, C.; Jiao, B.; Hou, X.; Duan, X.; $\mathrm{Wu}, \mathrm{Z}$. Charge transport between coupling colloidal perovskite quantum dots assisted by functional conjugated ligands. Angew. Chem. 2018, 130, 5856-5860.

(27) Wang, S.; Zhou, L.; Huang, F.; Xin, Y.; Jin, P.; Ma, Q.; Pang, Q.; Chen, Y.; Zhang, J. Z. Hybrid organic-inorganic lead bromide perovskite supercrystals self-assembled with Lcysteine and their highly luminescent properties. J. Mater. Chem. C. 2018, 6, 10994-11001.

(28) Zhou, M.; Ghosh, I.; Current Trends in Peptide Science Quantum Dots and Peptides: A Bright Future Together Biopolymers. Peptide Science. 2007, 88, 325-339

(29) Zhang, T.; Xie, L.; Chen, L.; Guo, N.; Li, G.; Tian, Z.; Mao, B.; and Zhao, Y.; In Situ Fabrication of Highly Luminescent, Bifunctional Amino Acid Crosslinked 2D/3D, $\mathrm{NH}_{3} \mathrm{C}_{4} \mathrm{H}_{9} \mathrm{COO}\left(\mathrm{CH}_{3} \mathrm{NH}_{3} \mathrm{PbBr}_{3}\right)$ n Perovskite Films, Adv. Funct. Mater. 2017, 27, 1603568

(30) Luo, B.; Bonabi Naghadeh, S.; Allen, A’L.; Li, X.; and Zhang, J. Z.; Peptide-Passivated Lead Halide Perovskite Nanocrystals Based on Synergistic Effect between Amino and Carboxylic Functional Groups, Adv. Funct. Mater. 2017, 27, 1604018

(31) Gonzalez-Carrero, S.; Martinez-Sarti, M.; Sessolo, M.; Galian, R. E.; Perez-Prieto, J. Highly photoluminescent, dense solid films from organic-capped $\mathrm{CH}_{3} \mathrm{NH}_{3} \mathrm{PbBr}_{3}$ perovskite colloids. J. Mater. Chem. C. 2018, 6, 6771-6777.

(32) Leyden, M. R.; Meng, L.; Jiang, Y.; Ono, L.K.; Qiu, L.; Juarez-Perez, E.J.; Qin, Ch.; Adachi, Ch.; and Qi, Y.; Methylammonium Lead Bromide Perovskite Light-Emitting Diodes by Chemical Vapor Deposition, J. Phys. Chem. Lett., 2017, 8, 3193-3198,

(33) Boix, P.P.; Agarwala, S.; Ming Koh, T.; Mathews, N.; and Mhaisalkar, S.G.; Perovskite Solar Cells: Beyond Methylammonium Lead Iodide, J. Phys. Chem. Lett., 2015, 6, 898-907 
(34) Kim, Y.; Yassitepe, E.; Voznyy, O.; Comin, R.; Walters, G.; Gong, X.; Kanjanaboos, P.; Nogueira, A. F.; and Sargent, E,H.; Efficient Luminescence from Perovskite Quantum Dot Solids, ACS Appl. Mater. Interfaces, 2015, 7, 25007-25013

(35) Rahimnejad, S.; Kovalenko, A.; Forés, S, M.; Aranda, C.; Guerrero, A.; Coordination Chemistry Dictates the Structural Defects in Lead Halide Perovskites, ChemPhysChem, 2016, 17, 2795-2798

(36) Yufit, D. S. and Howard, J. A. K.; Low-melting molecular complexes of chloroform, CrystEngComm, 2010,12, 737-741

(37) Parveen, A.; Sughanya, V.; Nagarajan, S.; Quenching of fluorescence in C60 fulleropyrrolidines by chloroform, Spectrochimica Acta Part A, 2016, 152, 77-81

(38) Saperstein, D.; Levin, E.; Fluorescence quenching of ultraviolet excited aromatic solutions by chloroform and several related chlorinated methanes, J. Chem. Phys. 1975, 62, 3560

(39) Kovalenko, A.; Yumusak, C.; Heinrichova, P.; Stritesky, S.; Fekete, L.; Vala, M.; Weiter, M.; Sariciftci N. S.; and Krajcovic, J.; Adamantane substitutions: a path to high-performing, soluble, versatile and sustainable organic semiconducting materials, J. Mater. Chem. C, 2017, 5, 4716-4723,

(40) Krajčovič, J.; Kovalenko, A.; Heinrichová, P.; Vala, M.; Weiter, M., Adamantyl side groups boosting the efficiency and thermal stability of organic solid-state fluorescent dyes, Journal of Luminescence, 2016, 175, 94-99

(41) Nenon, D. P.; Christians, J. A.; Wheeler, L. M.; Blackburn, J. L.; Sanehira, E. M.; Dou, B.; Olsen, M. L.; Zhu, K.; Berry J. J.; and Luther, J. M.; Structural and chemical evolution of methylammonium lead halide perovskites during thermal processing from solution, Energy Environ. Sci., 2016, 9, 2072-2082

(42) Wang, K.-H.; Li, L.-C.; Shellaiah, M.; Wen Sun, K. Structural and photophysical properties of methylammonium lead tribromide (MAPbBr3) single crystals. Sci. Rep. 2017, 7, 1-14.

(43) Maxwell, C. R.; Peterson, D. C.; Sharpless, N. E. The effect of ionizing radiation on amino acids. The effect of $\mathrm{x}$-rays on aqueous solutions of glycine. Radiat. Res. 1954, 1, 530-545.

(44) Zubavichus, Y.; Fuchs, O.; Weinhardt, L.; Heske, C.; Umbach, E.; Denlinger, J. D.; Grunze, M. Soft X-ray induced decomposition of amino acids: an XPS, mass spectrometry, and NEXAFS study. Radiat. Res. 2004, 161, 346-358.

(45) Sun, H.; Yang, Z.; Wie, M.; Sun, W.; Li, X.; Ye, S.; Zhao, Y.; Tan, H.; Kynaston, E. L.; Schon, T. B.; Yan, H.; Lu, Z.-H.; Ozin, G. A.; Sargent, E. H.; Seferos, D. S.; Chemically addressable perovskite nanocrystals for light-emitting applications. Adv. Mater. 2017, 29, 1701153.

(46) Ebrahiminezhad, A.; Ghasemi, Y.; Rasoul-Amini, S.; Barar, J.; Davaran, S., Impact of Amino-Acid Coating on the Synthesis and Characteristics of Iron-Oxide Nanoparticles (IONs), B. Korean Chem. Soc. 2012, 33, 3957-3962

(47) Carneiro, J.; Doll-Boscardin, P. M.; Fiorin, B. C.; Nadal, J. M.; Farago, P. V.; Paula, J. P. Development and characterization of hyaluronic acid-lysine nanoparticles with potential as innovate dermal filling. Braz. J. Pharm. Sci. 2016, 52, 645-651.

(48) Li, W.-C.; Lee, S.-W. The characteristics of lysine-mediated self-assembly of gold nanoparticles on the ITO glass. Curr. Appl. Phys. 2012, 12, 1361-1365.

(49) Duru, I.; Ege, D. Self-assembly of L-arginine on electrophoretically deposited hydroxyapatite coatings. ChemistrySelect. 2018, 3, 9041-9045.

(50) Goyal, R. N.; Bishnoi, S.; Chasta, H.; Abdul Aziz, M.; Oyama, M. Effect of surface modification of indium tin oxide by nanoparticles on the electrochemical determination of tryptophan. Talanta. 2011, 85, 2626-2631. 
(51) Havare, A. K.; Can, M.; Demic, S.; Okur, S.; Kus, M.; Aydin, H.; Yagmurcukardes, N.; Tari, S. Modification of ITO surface using aromatic small molecules with carboxylic acid groups for OLED applications. Synth. Met. 2011, 161, 2397-2404.

(52) Mali, S. S.; Shim, C.S.; Hong, C. K. Highly stable and efficient solid-state solar cells based on methylammonium lead bromide $\left(\mathrm{CH}_{3} \mathrm{NH}_{3} \mathrm{PbBr}_{3}\right)$ perovskite quantum dots. NPG Asia Materials. 2015, 7, 208.
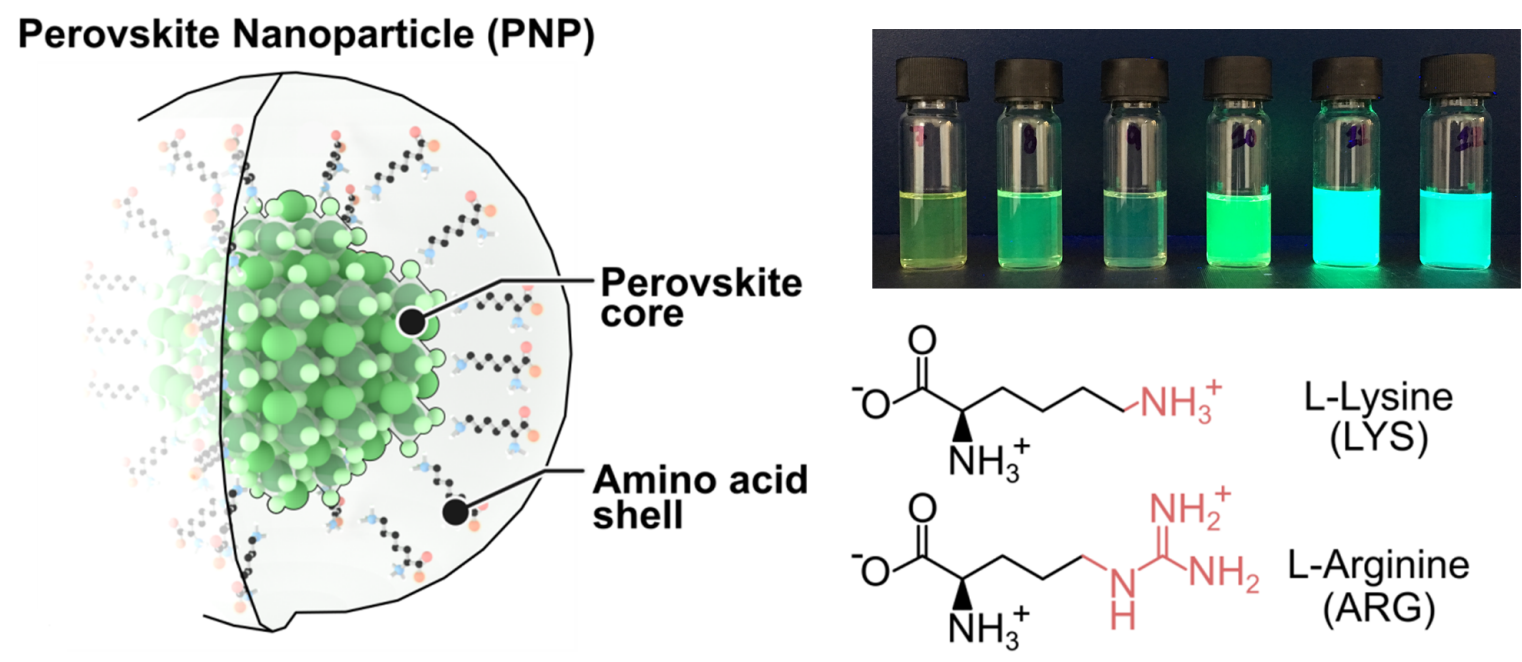